\title{
NEW PRACTICAL MODEL FOR SAND TRANSPORT INDUCED BY NON-BREAKING WAVES AND CURRENTS
}

\author{
Dominic A. van der A', Jan S. Ribberink², Jebbe J. van der Werf' and Tom O’Donoghue ${ }^{1}$
}

\begin{abstract}
Many existing practical sand transport formulae for the coastal marine environment are restricted to limited ranges of hydrodynamic and sediment conditions. This paper presents a new practical formula for net sand transport induced by non-breaking waves and currents, and currents alone. The formula is based on the semi-unsteady, half wave-cycle concept, with bed shear stress as the main forcing parameter. Unsteady phase-lag effects between velocities and concentrations are accounted for, which are especially important for rippled bed and fine sand sheet-flow conditions. Recently recognized effects on the net transport related to flow acceleration skewness and progressive surface waves are also included. The formula is calibrated against a large dataset of net transport rate measurements from oscillatory flow tunnels and a large wave flume covering a wide range of flow and sand conditions. Good agreement is obtained between observations and predictions, and its validity is shown for bedload dominated steady flow conditions.
\end{abstract}

Keywords: sediment transport formula; sheet flow, ripples; oscillatory flow; non-breaking waves

\section{INTRODUCTION}

Sand transport models for the coastal marine environment are generally empirical formulas based on experiments with specific ranges of hydrodynamic and sediment conditions (e.g. Bailard 1981; Dibajnia and Watanabe 1992; Ribberink 1998; Nielsen 2006; Van Rijn 2007). For example, many models have focused on velocity-skewed $\left(2^{\text {nd }}\right.$ order Stokes) waves and do not take into account the transport effects resulting from acceleration skewness (sawtooth shaped waves). Other models which are specifically developed for high energy flat bed sheet-flow conditions are not able to predict transport rates under more moderate conditions when the bed is generally covered with ripples. Furthermore, models that are based on a quasi-steady assumption between the transport and velocity or bed shear stress are not able to predict transport rates that are affected by phase differences between the velocity and concentration fields. Under certain conditions, these so-called "phase lag effects" can dominate the transport, both in the rippled bed and sheet flow regimes. Moreover, nearly all the conventional "wave-induced" sand transport models can essentially only be applied to horizontally uniform oscillatory flows; they do not account for effects related to progressive surface waves that further influence the net transport (Ribberink et al. 2000).

The limited capabilities of many existing models are, to some extent, the result of the limited range of conditions contained in the dataset that have been used for the development of these models. For this reason Van der Werf et al. (2009) brought together existing transport rate measurements from a number of facilities covering a wide range of wave and wave-current conditions. The database has recently been significantly extended with new transport data for acceleration-skewed oscillatory flows (Van der A et al. 2010; Silva et al. submitted) and data for progressive surface waves obtained in a large wave flume (Schretlen et al. 2009). This database is used for the development and calibration of the present practical sand transport model.

This work has been part of the SANTOSS collaborative research project. Initial stages of the development of the present transport model were reported by Van der Werf et al. (2007). This paper presents the final model developed during the SANTOSS project. Model development has specifically focused on including the effects of acceleration skewness and progressive surface waves. The paper is organized as follows. In the next section the net transport formula is presented, with subsections dedicated to the acceleration skewness and progressive surface wave effects. The following section illustrates the performance of the new formula, including its capabilities to predict transport rates for steady flow conditions. The conclusions are presented in the final section.

\footnotetext{
${ }^{1}$ School of Engineering, University of Aberdeen, AB24 3UE, Aberdeen, UK

${ }^{2}$ Water Engineering and Management, University of Twente, PO Box 143, Enschede, The Netherlands

${ }^{3}$ Marine and Coastal Systems, Deltares (formerly Delft Hydraulics), P.O. Box 177, 2600 MH, Delft, The Netherlands
} 


\section{MODEL}

\section{Model concept}

The net transport model is based on a modified version of the "half-cycle" model concept initially proposed by Dibajnia and Watanabe (1992). In this concept the wave-averaged transport rate is essentially the difference between the amount of sand transported during the positive "crest" half cycle and the amount of sand transported during the negative "trough" half cycle. Unsteady phase lag effects are taken into account by accounting for two contributions to the amount of sand transport during each half cycle: sand entrained and transported during the present half cycle and sand entrained during the previous half cycle which is transported during the present half cycle, the latter being the phase lag contribution. Each half cycle, the magnitude of the phase lag contribution is governed by a phase lag parameter which is the ratio of the fall time of the sand particles and the period of the corresponding half cycle, and thus represents the tendency for a particle to remain in suspension. The main driving mechanism in Dibajnia and Watanabe's (1992) model is the near bed (free-stream) velocity.

The new model proposed in the remainder of this paper includes the following main modifications to Dibajnia and Watanabe's (1992) original formulation:

- $\quad$ bed shear stress is the main driving parameter;

- the phase lag effects related to flow unsteadiness are modeled in a different way;

- the effects of the wave shape (velocity- and acceleration skewness) are incorporated;

- $\quad$ effects specifically related to progressive surface waves are accounted for;

- the model is capable of dealing with waves and currents under an angle.

These new modifications will be presented in the three sections. First a description of the "basic" model will be given, capable of dealing with velocity-skewed flows and currents, followed by a section on the additional modifications regarding acceleration skewness. The last section presents the modifications related to progressive surface waves. Although the complete model is able to deal with waves and currents under arbitrary angle, for brevity reasons we will focus in the present paper only on waves and collinear currents. For the complete description of the new formula in vector notation, see Ribberink et al. (2010).

\section{Model Formulation for Velocity-Skewed Flow plus Current}

Fig. 1 illustrates a near-bed velocity time-series at the edge of the wave boundary layer due to combined wave-current motion:

$$
u(t)=u_{\delta}+\tilde{u}(t)
$$

with $u_{\delta}$ the net current at $z=\delta$ calculated from the measured current velocity by assuming a logarithmic velocity profile; in the present model we have taken $\delta=0.2 \mathrm{~m}$. Furthermore it is assumed that the orbital velocity time-series, $\tilde{u}(t)$, and therefore the velocity characteristics $\left(\tilde{u}_{\mathrm{c}}, \tilde{u}_{\mathrm{t}}, \tilde{u}_{\mathrm{rms}} T_{\mathrm{c}}, T_{\mathrm{t}}, T_{\mathrm{cu}}, T_{\mathrm{tu}}\right)$, are known a priori. A characteristic orbital velocity amplitude $\hat{u}$ and characteristic orbital excursion amplitude $a$ for the complete wave cycle are calculated from:

$$
\begin{gathered}
\hat{u}=\sqrt{2} \tilde{u}_{\mathrm{rms}} \\
\hat{a}=\frac{\hat{u} T}{2 \pi}
\end{gathered}
$$

For the purpose of calculating the shear stress each half cycle (see next section) we use a representative orbital velocity for the wave crest and wave trough, defined as the root-mean-square velocity assuming a sinusoidal flow:

$$
\begin{aligned}
& \tilde{u}_{\mathrm{cr}}=\frac{1}{2} \sqrt{2} \tilde{u}_{\mathrm{c}} \\
& \tilde{u}_{\mathrm{tr}}=\frac{1}{2} \sqrt{2} \tilde{u}_{\mathrm{t}}
\end{aligned}
$$

The characteristic half-cycle velocities in the case of an oscillatory flow with current now become: 


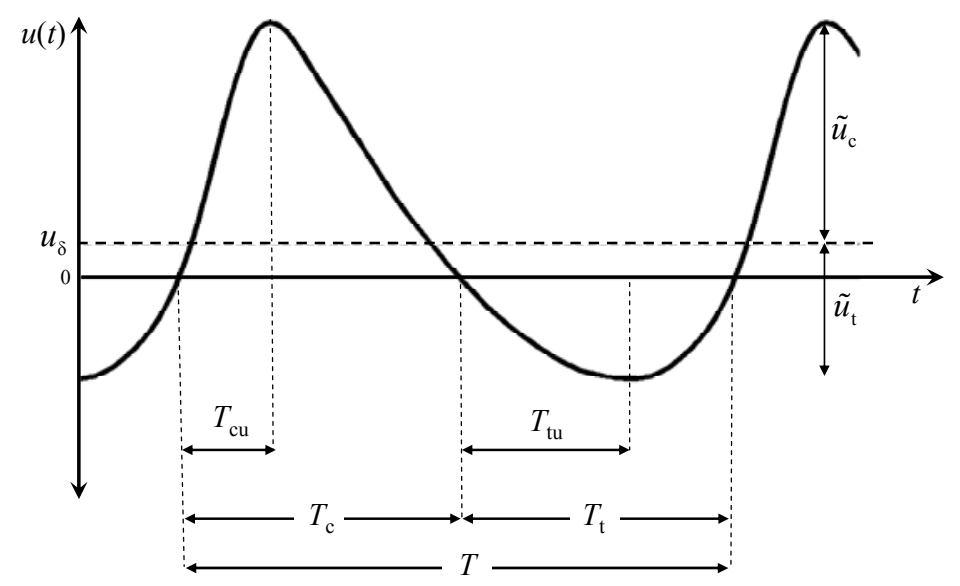

Figure 1. Example velocity time-series used as input to the formula. The subscripts "c" and " $t$ " represent the crest and trough direction respectively.

$$
\begin{aligned}
& u_{\mathrm{cr}}=\tilde{u}_{\mathrm{cr}}+u_{\delta} \\
& u_{\mathrm{tr}}=-\tilde{u}_{\mathrm{tr}}+u_{\delta}
\end{aligned}
$$

The degree of velocity skewness is expressed through the parameter $R$ :

$$
R=\frac{\tilde{u}_{\mathrm{c}}}{\tilde{u}_{\mathrm{c}}+\tilde{u}_{\mathrm{t}}}
$$

Similarly, the degree of acceleration skewness is expressed through the parameter $\beta$ for which:

$$
\beta=\frac{\tilde{\dot{u}}_{\mathrm{c}}}{\tilde{\dot{u}}_{\mathrm{c}}+\tilde{\dot{u}}_{\mathrm{t}}}
$$

with $\tilde{\dot{u}}_{\mathrm{c}}$ and $\tilde{\dot{u}}_{\mathrm{t}}$ the amplitudes of the horizontal flow acceleration in crest and trough direction respectively. For the conditions presented in the present paper $R$ and $\beta$ were known from measurements, and therefore the periods $T_{\mathrm{c}}, T_{\mathrm{t}}, T_{\mathrm{cu}}, T_{\mathrm{tu}}$ could be determined from standard shapes for velocity skewness ( $2^{\text {nd }}$ order Stokes) and acceleration skewness (sawtooth time-series, see Ribberink et al. 2010). However, for wider application the hydrodynamic input parameters need to be derived from local wave parameters and bathymetry. It is suggested to use the set of empirical formulae proposed by Elfrink et al. (2006), which enables the calculation of the near bed velocity time-series, including velocity and acceleration skewness, based on the wave height and period, water depth, and local bed slope.

The dimensionless net transport rate is given by:

$$
\Phi=\frac{q_{\mathrm{s}}}{\sqrt{(s-1) g d_{50}^{3}}}=\frac{\sqrt{\left|\theta_{\mathrm{c}}\right|} T_{\mathrm{c}}\left(\Omega_{\mathrm{cc}}+\Omega_{\mathrm{tc}}\right) \frac{\theta_{\mathrm{c}}}{\left|\theta_{\mathrm{c}}\right|}+\sqrt{\left|\theta_{\mathrm{t}}\right|} T_{\mathrm{t}}\left(\Omega_{\mathrm{tt}}+\Omega_{\mathrm{ct}}\right) \frac{\theta_{\mathrm{t}}}{\left|\theta_{\mathrm{t}}\right|}}{T}
$$

where $q_{\mathrm{s}}$ is the volumetric net transport rate per unit width, $s=\rho_{\mathrm{s}} / \rho$ where $\rho_{\mathrm{s}}$ and $\rho$ are the densities of sand and water respectively, $g$ is acceleration due to gravity, $d_{50}$ is the sand median diameter and $\theta$ the Shields parameter. There are four contributions to the net sand transport:

- $\Omega_{\mathrm{cc}}$ represents the sand load that is entrained during the wave crest period and transported during the crest period,

- $\Omega_{\mathrm{ct}}$ represents the sand load that is entrained during the wave crest period and transported during the trough period,

- $\Omega_{\mathrm{tt}}$ represents the sand load that is entrained during the wave trough period and transported during the trough period, 
- $\Omega_{\mathrm{tc}}$ represents the sand load that is entrained during the wave trough period and transported during the crest period

These half-cycle sand loads are multiplied by $\sqrt{\theta}$, representing a (non-dimensional) near-bed (friction) velocity, to obtain a non-dimensional half-cycle transport rate. Both half-cycle transport rates are weighted with their duration relative to the wave period ( $T_{\mathrm{c}} / T$ and $T_{\mathrm{t}} / T$, respectively).

The load contributions are calculated in the following manner:

$$
\begin{gathered}
\Omega_{\mathrm{cc}}= \begin{cases}\Omega_{\mathrm{c}} & \text { if } \quad P_{\mathrm{c}} \leq 1 \\
\frac{1}{P_{\mathrm{c}}} \Omega_{\mathrm{c}} & \text { if } \quad P_{\mathrm{c}}>1\end{cases} \\
\Omega_{\mathrm{ct}}= \begin{cases}0 & \text { if } \quad P_{\mathrm{c}} \leq 1 \\
\frac{\left(P_{\mathrm{c}}-1\right)}{P_{\mathrm{c}}} \Omega_{\mathrm{c}} & \text { if } \quad P_{\mathrm{c}}>1\end{cases} \\
\Omega_{\mathrm{tt}}=\left\{\begin{array}{lll}
\Omega_{\mathrm{t}} & \text { if } \quad P_{\mathrm{t}} \leq 1 \\
\frac{1}{P_{\mathrm{t}}} \Omega_{\mathrm{t}} & \text { if } \quad P_{\mathrm{t}}>1
\end{array}\right. \\
\Omega_{\mathrm{tc}}= \begin{cases}0 & \text { if } P_{\mathrm{t}} \leq 1 \\
\frac{\left(P_{\mathrm{t}}-1\right)}{P_{\mathrm{t}}} \Omega_{\mathrm{t}} & \text { if } \quad P_{\mathrm{t}}>1\end{cases}
\end{gathered}
$$

Thus, in the equations above when the phase lag parameter $P$ exceeds the critical value of 1 , there is an exchange of sand from one half cycle to the next. The sand load entrained in the flow during each half cycle depends on the Shields parameter as follows (with subscript " $i$ " either "c" for crest or " $t$ " for trough):

$$
\Omega_{i}= \begin{cases}0 & \text { if }\left|\theta_{i}\right| \leq \theta_{\mathrm{cr}} \\ m\left(\left|\theta_{i}\right|-\theta_{\mathrm{cr}}\right)^{n} & \text { if }\left|\theta_{i}\right|>\theta_{\mathrm{cr}}\end{cases}
$$

where the critical Shields number, $\theta_{\mathrm{cr}}$, is calculated following Soulsby (1997) and the coefficients $m$ and $n$ are calibration coefficients. The phase lag parameter $P_{i}$ represents the ratio of a representative sediment stirring height and the sediment settling distance:

$$
P_{i}= \begin{cases}\alpha_{\mathrm{r}} \frac{\eta}{T_{i} w_{s}} & \text { if } \eta>0 \text { (ripple regime) } \\ \alpha_{\mathrm{s}} \frac{\delta_{\mathrm{s} i}}{T_{i} w_{s}} & \text { if } \eta=0 \text { (sheet flow regime) }\end{cases}
$$

where $\eta$ is the ripple height and $\delta_{\mathrm{s} i}$ the sheet flow layer thickness. The sediment settling velocity, $w_{\mathrm{s}}$, is calculated according to Soulsby's (1997) method assuming a grain size of suspended sand $d_{\mathrm{s}}=0.8 d_{50}$. The coefficients $\alpha_{\mathrm{s}}$ and $\alpha_{\mathrm{r}}$ are used for model calibration, as described hereafter.

The Shields parameter for oscillatory flow plus current is calculated following:

$$
\theta_{i}=\frac{\frac{1}{2} f_{\mathrm{w} \delta} u_{i \mathrm{r}}\left|u_{i \mathrm{r}}\right|}{(s-1) g d_{50}}
$$

Following Madsen and Grant (1976) the friction factor $f_{\mathrm{w} \delta i}$ is calculated as a linear combination of the wave friction $f_{\mathrm{w} i}$ factor and the current friction factor $f_{\delta}$ : 


$$
f_{\mathrm{w} \delta}=\alpha f_{\delta}+(1-\alpha) f_{\mathrm{w}}
$$

with

$$
\alpha=\frac{\sigma\left|u_{\delta}\right|}{\sigma\left|u_{\delta}\right|+\hat{u}}
$$

where $\sigma=3$ is a calibration factor. The wave friction factor is defined following Swart (1974):

$$
\begin{array}{llll}
f_{\mathrm{w}}=0.00251 \exp \left[5.21\left(\frac{\hat{a}}{k_{\mathrm{sw}}}\right)^{-0.19}\right] & \text { for } & \frac{\hat{a}}{k_{\mathrm{sw}}}>1.587 \\
f_{\mathrm{w}}=0.3 & \text { for } & \frac{\hat{a}}{k_{\mathrm{sw}}} \leq 1.587
\end{array}
$$

The wave roughness height $k_{\mathrm{sw}}$ includes the mobile bed roughness for sheet flow conditions and an additional form roughness in the presence of ripples:

$$
k_{\mathrm{sw}}=\max \left\{d_{50}, d_{50}[\mu+6(\langle|\theta|\rangle-1)]\right\}+p \cdot \eta^{2} / \lambda
$$

with $\lambda$ is the ripple length and $p$ a calibration parameter $p=0.4$. In the case of sheet flow conditions the bed roughness is solved iteratively, since the mean absolute Shields parameter $\langle|\theta|\rangle$ depends on the bed roughness $k_{\mathrm{sw}}$ (Ribberink, 1998). The parameter $\mu$ is introduced to create an increased roughness and sand load for fine sand conditions $\left(d_{50}<0.15 \mathrm{~mm}\right)$ and results from calibration tests. For fine sands $\mu=6$ which linearly reduces to $\mu=1$ for medium and coarser sands $\left(d_{50}>0.20 \mathrm{~mm}\right)$. Using $\mu=6$ the predicted roughness heights for fine sands still fall within the range of measured roughness heights by Wilson (1989) which formed the basis of Ribberink's (1998) formulation (for more details see Ribberink et al., 2010).

The current-related friction factor is calculated assuming a logarithmic velocity profile:

$$
f_{\delta}=2\left[\frac{0.4}{\ln \left(30 \delta / k_{\mathrm{s} \delta}\right)}\right]^{2}
$$

in which the current-related roughness $\mathrm{k}_{\mathrm{s} \delta}$ is calculated in a similar way as in Eq. 21:

$$
k_{\mathrm{s} \delta}=\max \left\{3 d_{90}, d_{50}[\mu+6(\langle|\theta|\rangle-1)]\right\}+p . \eta^{2} / \lambda
$$
(1999):

Finally, the sheet flow layer thickness $\delta_{\mathrm{s} i}$ in Eq. 16 is calculated following Dohmen-Janssen

$$
\frac{\delta_{\mathrm{s} i}}{d_{50}}= \begin{cases}25\left|\theta_{\mathrm{w} i}\right| & \text { if } d_{50} \leq 0.15 \mathrm{~mm} \\ {\left[25+\left(10^{3} d_{50}-0.15\right) \frac{(13-25)}{(0.20-0.15)}\right]\left|\theta_{\mathrm{w} i}\right|} & \text { if } 0.15 \mathrm{~mm}<d_{50}<0.20 \mathrm{~mm} \\ 13\left|\theta_{\mathrm{w} i}\right| & \text { if } d_{50} \geq 0.20 \mathrm{~mm}\end{cases}
$$

This equation differs slightly from Dohmen-Janssen's (1999) original formulation since the multiplier for fine sands is reduced from 35 in the original formulation to 25 in Eq. 24 in order to compensate for the increased mobile bed roughness for fine sands in the sheet-flow regime.

In the development and calibration of the model using laboratory data, the measured ripple dimensions were used as input to avoid any uncertainty related to applying a ripple predictor. In all other applications of the model the ripple dimensions are predicted using the ripple predictor of O'Donoghue et al. (2006), in which the ripple height and ripple length are a function of the grain size $d_{50}$ and maximum mobility number $\Psi_{\max }\left(=\tilde{u}_{\mathrm{c}} /(s-1) g d_{50}\right)$. In the present model the ripple predictor is adjusted to allow for a smooth transition between the roughness in the ripple regime $\left(\Psi_{\max }<190\right)$ and the sheet flow regime $\left(\Psi_{\max } \geq 240\right)$. 

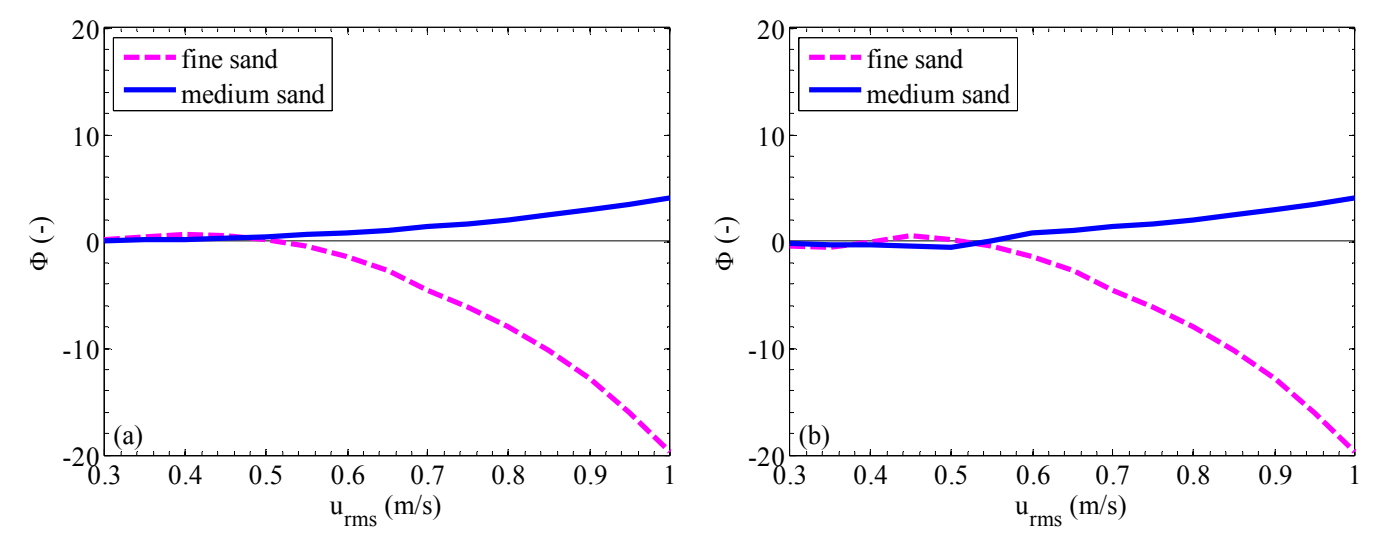

Figure 2: Non-dimensional transport rate as function of $u_{\mathrm{rms}}$ for a velocity-skewed oscillatory flow with $T=$ $6.5 \mathrm{~s}, R=0.62$ and $\beta=0.5$ and fine sand with $d_{50}=0.13 \mathrm{~mm}$ and medium sand with $d_{50}=0.25 \mathrm{~mm}$ : (a) flat bed conditions; (b) with ripple predictor switched on.

Fig. 2 illustrates the "behaviour" of the predicted (non-dimensional) transport rates with $u_{\mathrm{rms}}$ for a typical fine and medium sand. To highlight the effect of ripples on the transport rates, Fig. 2a shows the predictions by assuming a flat bed over the entire $u_{\text {rms }}$ range (ripple predictor switched off), while in Fig. $2 b$ the ripple regime is included in the transport rate predictions (ripple predictor switched on). Fig. 2a shows that for medium sand the transport shows a quasi-steady behaviour, i.e. increasing transport with increasing velocity. For fine sand on the other hand, phase-lags effects in the sheet-flow layer dominate the transport rate, which changes direction and becomes increasingly larger in the negative direction with increasing velocity. Introducing ripples changes the transport rate direction in the lower $u_{\text {rms }}$ range for medium and fine sands, since for both sands ripple-related phase-lag effects dominate the net transport. The behaviour illustrated in Fig. $2 b$ is in agreement with observations from laboratory experiments as may be inferred from Van der Werf et al. (2009).

\section{Acceleration skewness effects}

For oscillatory flows with acceleration skewness the following additional transport mechanisms are included in the model:

1. Increased bed shear stress asymmetry with increasing acceleration skewness.

2. Increase/decrease of sediment settling time during the crest/trough period with increasing acceleration skewness.

3. Increase/decrease of the effective travel distance of the suspended load during crest/trough period.

Increasing acceleration skewness leads to larger peak bed shear stress under the strongly accelerating (crest) half-cycle, because the boundary layer has less time to develop before maximum velocity is reached. Accordingly, under the weakly accelerating (trough) half cycle the peak bed shear stress is reduced because the boundary layer has much more time to grow until maximum velocity is reached (Nielsen 1992; Van der A et al. 2008). This effect is included in the present model by defining separate friction factors for the wave crest and trough following the approach by Silva et al. (2006):

$$
\begin{aligned}
& f_{\mathrm{w} i}=0.00251 \exp \left[5.21\left(\frac{\left(\frac{2 T_{i \mathrm{u}}}{T_{i}}\right)^{2} \hat{a}}{k_{\mathrm{sw}}}\right)^{-0.19}\right] \quad \text { for } \quad \frac{\hat{a}}{k_{\mathrm{sw}}}>1.587 \\
& f_{\mathrm{w} i}=0.3 \quad \text { for } \quad \frac{\hat{a}}{k_{\mathrm{sw}}} \leq 1.587
\end{aligned}
$$

For example, for a forward-leaning crest half-cycle, $\left(2 T_{\mathrm{cu}} / T_{\mathrm{c}}\right)^{2}<1$, which leads to a larger friction factor and hence bed shear stress compared to the equivalent symmetric (sinusoidal) half cycle for 
which $\left(2 T_{\mathrm{cu}} / T_{\mathrm{c}}\right)^{2}=1$ (thus Eq. 25 reduces to Eq. 20). The choice of the power 2 is fairly arbitrary, however Van der A (2010) has shown that it yields very good agreement with the measurements of bed shear stress for acceleration-skewed flow. Incorporating this "enhanced" bed shear stress for acceleration-skewed flow affects two primary transport processes in the model. Firstly, even though the magnitude of the free-stream velocity is equal in both directions, an asymmetry is created in the amount of sand that is entrained each half wave cycle (Eq. 15). Secondly, because within the boundary layer the maximum velocity is larger under the crest half cycle compared to the trough half cycle (Van der A et al., 2008), the same amount of sand will travel further under the crest half cycle compared to the trough half cycle. This effect is implied in the transport equation (Eq. 27), since in the new model the travel distance is associated with the friction velocity $(\sim \sqrt{\theta})$ rather than the free-stream velocity.

To account for the increased settling time during the crest half cycle and decreased settling time during the trough half cycle caused by acceleration skewness, the phase lag parameter given by Eq. 16 is replaced by:

$$
P_{i}= \begin{cases}\alpha_{\mathrm{r}} \frac{\eta}{2\left(T_{i}-T_{i \mathrm{u}}\right) w_{s}} & \text { if } \eta>0 \text { (ripple regime) } \\ \alpha_{\mathrm{s}} \frac{\delta_{\mathrm{s} i}}{2\left(T_{i}-T_{i \mathrm{u}}\right) w_{s}} & \text { if } \eta=0 \text { (sheet flow regime) }\end{cases}
$$

thus recognising that with increasing (forward leaning) acceleration skewness the settling time during the crest half cycle increases, leading to smaller $P_{\mathrm{c}}$ and the settling time during the trough half cycle decreases, leading to larger $P_{\mathrm{t}}$. In case of no acceleration skewness $2\left(T_{\mathrm{c}}-T_{\mathrm{cu}}\right)=T_{\mathrm{c}}$ and $2\left(T_{\mathrm{t}}-T_{\mathrm{tu}}\right)=T_{\mathrm{t}}$ and Eq. 26 reduces to Eq. 16.

The amounts of sand that are carried over to the next half cycle, $\Omega_{\mathrm{tc}}$ and $\Omega_{\mathrm{ct}}$, are further affected by acceleration skewness, since the sand remaining in suspension after half a wave cycle is transported further when followed by a steep front half-wave cycle compared to a gradual front half-wave cycle, since for a given (short) duration after flow reversal, $\Delta t$, the travel distance $u \Delta t$ is larger. This effect is captured by increasing/decreasing the travel distance of the 'phase-lag' loads $\Omega_{\text {tc }}$ respectively $\Omega_{\mathrm{ct}}$ with increasing acceleration skewness. The transport equation is therefore extended as follows:

$$
\Phi=\frac{q_{\mathrm{s}}}{\sqrt{(s-1) g d_{50}^{3}}}=\frac{\sqrt{\left|\theta_{\mathrm{c}}\right|} T_{\mathrm{c}}\left(\Omega_{\mathrm{cc}}+\frac{T_{\mathrm{c}}}{2 T_{\mathrm{cu}}} \Omega_{\mathrm{tc}}\right) \frac{\theta_{\mathrm{c}}}{\left|\theta_{\mathrm{c}}\right|}+\sqrt{\left|\theta_{\mathrm{t}}\right|} T_{\mathrm{t}}\left(\Omega_{\mathrm{tt}}+\frac{T_{\mathrm{t}}}{2 T_{\mathrm{tu}}} \Omega_{\mathrm{ct}}\right) \frac{\theta_{\mathrm{t}}}{\left|\theta_{\mathrm{t}}\right|}}{T}(27)
$$

Again, when there is no acceleration skewness the effect disappears and Eq. 27 reverts back to Eq. 10 .

The transport behaviour for a typical purely acceleration-skewed flow (i.e. sawtooth shaped) is shown in Fig. 3. For flat bed conditions (Fig. 3a) both the fine and medium sand transport rates increase with increasing $u_{\mathrm{rms}}$, albeit that the fine sand transport rates are much larger compared to the medium sand transport rates. In part this is due to the enhanced roughness for fine sands, but the main mechanism responsible are the phase lag effects related to acceleration skewness, which now augment the positive net transport. This is in strong contrast to the velocity-skewed flows (Fig. 2), where the positive fine sand transport rates diminish and even become largely negative. Similarly, introducing ripples (Fig. 3b) increases the positive transport rates for both sands, again, in part due to the enhanced roughness but also due to ripple-related phase lag effects now augmenting the positive transport rates. Although no observations exist of transport rates by acceleration skewed flow in the ripple regime, the behaviour predicted by model and shown in Fig. 3 for both sand sizes in the sheet flow regime agrees well with the transport behaviour for acceleration skewed flows measured by Van der A et al. (2010). 

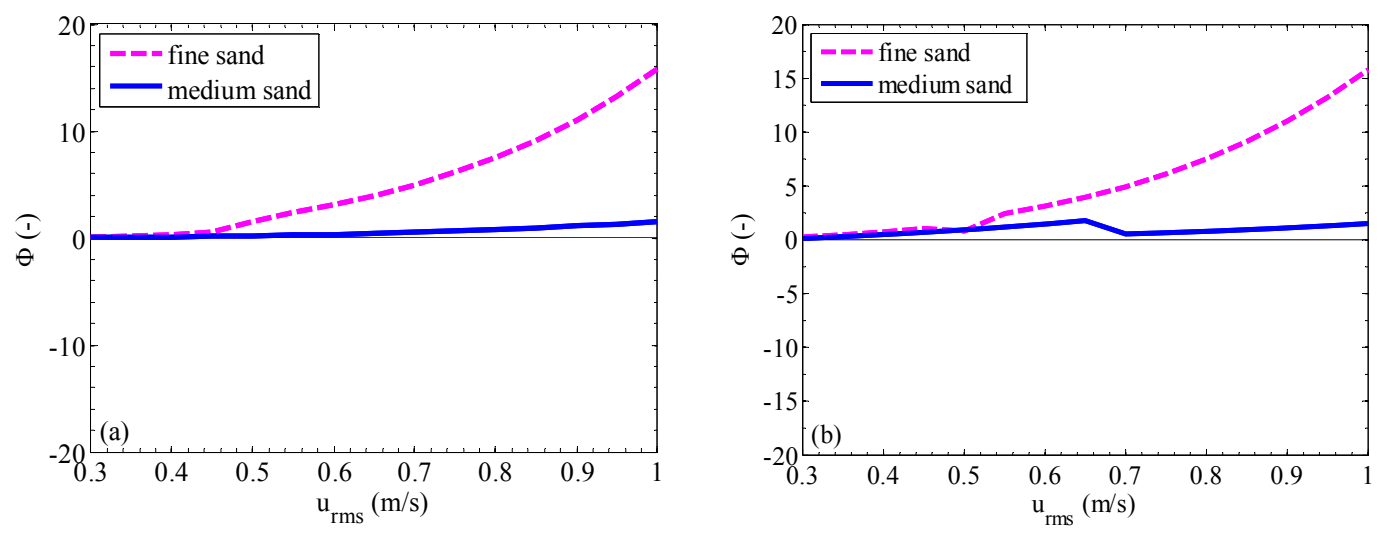

Figure 3: Non-dimensional transport rate as function of $u_{\mathrm{rms}}$ for a acceleration-skewed oscillatory flow with $T$ $=6.5 \mathrm{~s}, \beta=0.75, R=0.5$ : (a) flat bed conditions; (b) with ripple predictor switched on.

\section{Surface waves}

For progressive surface waves the influence of three additional flow mechanisms is brought into the transport model:

1. The wave-Reynolds stress,

2. The Lagrangian grain motion, which differs during the crest and trough period.

3. The vertical orbital velocity affecting the grain settling velocity near the bed.

Following the approach as suggested by Nielsen (2006), the wave-Reynolds stress is added as additional (onshore) component to the Shields parameter for the wave crest and wave trough (subscript "sw" denotes surface waves):

$$
\theta_{i, \mathrm{sw}}=\theta_{i}+\theta_{\mathrm{wRe}}
$$

The "wave Reynolds" Shields parameter, $\theta_{\mathrm{wRe}}$, accounts for the streaming related bed shear stress as follows:

$$
\theta_{\mathrm{wRe}}=\frac{\tau_{\mathrm{wRe}}}{\rho(s-1) g d_{50}}
$$

with the wave Reynolds stress following Nielsen (2006):

$$
\tau_{\mathrm{wRe}}=\rho \frac{f_{\mathrm{w} \delta}}{2 c} \alpha_{\mathrm{w}} \hat{u}^{3}
$$

in which $\alpha_{\mathrm{w}}=4 /(3 \pi)=0.424$ and $c$ is the wave speed.

This additional stress enhances the crest shear stress but reduces the trough shear stress and thus has a similar influence as an increase in wave asymmetry (velocity skewness).

For surface waves sediment grains move with the wave during the wave crest and against the wave during the wave trough (Lagrangian motion). In this way they experience a longer crest period $T_{\mathrm{c}, \mathrm{sw}}(=$ $\left.T_{\mathrm{c}}+\Delta T_{\mathrm{c}}\right)$ and therefore a longer onshore travel distance, but also a shorter trough period $T_{\mathrm{t}, \mathrm{sw}}\left(=T_{\mathrm{t}}-\right.$ $\Delta T_{\mathrm{t}}$ ) and thus a shorter offshore travel distance. As a result this will lead to an increase of the net sand transport in the direction of wave propagation (generally onshore).

$\Delta T$ depends on the ratio of the wave speed $c$ and the horizontal grain displacement during the half wave-cycle (orbital diameter) $d_{\mathrm{g}}$ :

$$
\Delta T=\frac{d_{\mathrm{g}}}{c}
$$

Assuming a sinusoidal wave shape for the half-cycle horizontal grain motion, the crest-period extension can be written as follows (for the derivation see Ribberink et al., 2010): 


$$
\Delta T_{\mathrm{c}}=\frac{d_{g}}{c}=\left\{\frac{c}{\zeta \hat{u}} \pi-2\right\}^{-1} T
$$

During the wave trough, the period and orbital diameter are reduced and the following expression follows:

$$
\Delta T_{\mathrm{t}}=\frac{d_{g}}{c}=\left\{\frac{c}{\zeta \hat{u}} \pi+2\right\}^{-1} T
$$

herein $\zeta$ is the ratio of the horizontal grain-velocity amplitude and the free-stream velocity amplitude. The magnitude of this ratio is estimated from detailed velocity measurements in the sheet flow layer carried out under full-scale surface waves in a large wave flume (GWK, Hannover). It is shown for a range of conditions that the ratio is more or less constant and can be roughly estimated as $\zeta=0.55$ (see Ribberink et al. 2010). This value is adopted in the present model. The (Lagrangian) half-cycle duration in the case of surface waves are now calculated using:

$$
\begin{aligned}
& T_{\mathrm{c}, \mathrm{sw}}=T_{\mathrm{c}}+\Delta T_{\mathrm{c}} \\
& T_{\mathrm{t}, \mathrm{sw}}=T_{\mathrm{t}}-\Delta T_{\mathrm{t}}
\end{aligned}
$$

For surface waves a vertical orbital velocity is present which may affect the settling of grains. Although this velocity is small near the bed, it can still be of the order of magnitude of the settling velocity, especially for fine sand and high waves. In those conditions the vertical orbital motion therefore directly influences the phase lag process (see also Kranenburg et al. 2010). In the present transport model we include this effect by enhancing the settling velocity of the crest sediment load and reducing it for the trough sediment load. In the deceleration phase of the crest flow the settling process dominates and is magnified by the presence of a downward vertical orbital flow. Similarly the settling of the trough load is reduced due to the presence of an upward vertical orbital flow. We use non-linear $\left(2^{\text {nd }}\right.$-order Stokes) wave theory to determine the vertical orbital velocity amplitude at elevation $z$ near the bed $\hat{w}(z)$. The sand settling velocities at crest and trough are now corrected with the vertical velocity at level $r_{\mathrm{i}}$ as follows:

$$
\begin{gathered}
w_{\mathrm{sc}}=w_{\mathrm{s}}+\hat{w}\left(r_{\mathrm{c}}\right) \\
w_{\mathrm{st}}=\max \left(w_{\mathrm{s}}-\hat{w}\left(r_{\mathrm{t}}\right), 0\right)
\end{gathered}
$$

with

$$
r_{i}= \begin{cases}\varepsilon \eta & \text { if } \eta>0 \text { (ripple regime) } \\ \varepsilon \delta_{\mathrm{s} i} & \text { if } \eta=0 \text { (sheet flow regime) }\end{cases}
$$

Note that the trough settling velocity, $w_{\text {st }}$, is not allowed to become negative, its minimum is therefore limited to zero. The factor $\varepsilon$ is a calibration factor; in the calibrated model $\varepsilon=3$. Incorporating Eqs. 3437 in the phase lag parameter results in:

$$
P_{i}= \begin{cases}\alpha_{\mathrm{r}} \frac{\eta}{2\left(T_{i, \mathrm{sw}}-T_{i \mathrm{u}, \mathrm{sw}}\right) w_{\mathrm{s} i}} & \text { if } \eta>0 \text { (ripple regime) } \\ \alpha_{\mathrm{s}} \frac{\delta_{\mathrm{s} i}}{2\left(T_{i, \mathrm{sw}}-T_{i \mathrm{u}, \mathrm{sw}}\right) w_{\mathrm{s} i}} & \text { if } \eta=0 \text { (sheet flow regime) }\end{cases}
$$

The crest/trough period correction as well as the settling velocity correction have a similar influence, i.e. a reduction of $P_{\mathrm{c}}$ and an increase of $P_{\mathrm{t}}$, both leading to a (further) shift of the mean sand transport in onshore direction. 
The net transport rate now becomes:

$$
\Phi=\frac{q_{\mathrm{s}}}{\sqrt{(s-1) g d_{50}^{3}}}=\frac{\sqrt{\left|\theta_{\mathrm{c}, \mathrm{sw}}\right|} T_{\mathrm{c}, \mathrm{sw}}\left(\Omega_{\mathrm{cc}}+\frac{T_{\mathrm{c}, \mathrm{sw}}}{2 T_{\mathrm{cu}, \mathrm{sw}}} \Omega_{\mathrm{tc}}\right) \frac{\theta_{\mathrm{c}, \mathrm{sw}}}{\left|\theta_{\mathrm{c}, \mathrm{sw}}\right|}+\sqrt{\left|\theta_{\mathrm{t}, \mathrm{ww}}\right|} T_{\mathrm{t}, \mathrm{sw}}\left(\Omega_{\mathrm{tt}}+\frac{T_{\mathrm{t}, \mathrm{sw}}}{2 T_{\mathrm{tu}, \mathrm{sw}}} \Omega_{\mathrm{ct}}\right) \frac{\theta_{\mathrm{t}, \mathrm{sw}}}{\left|\theta_{\mathrm{t}, \mathrm{sw}}\right|}}{T}
$$

Note that when the flow is horizontally uniform oscillatory flow, as in a flow tunnel, the flow is essentially a wave with infinite wave length and wave speed and Eq. 40 reverts to Eq. 27 since $c \rightarrow \infty$ and $\hat{w}(z)=0$.
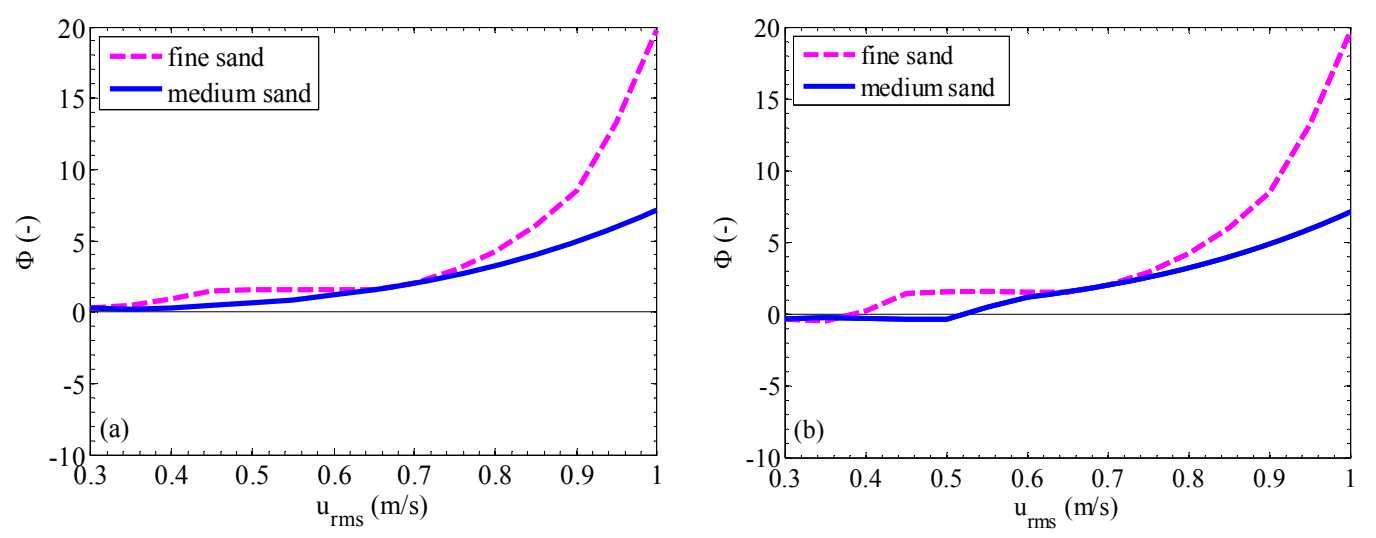

Figure 4: Non-dimensional transport rate as function of $u_{\mathrm{rms}}$ for a progressive surface wave with $T=6.5 \mathrm{~s}, R=$ $0.62, \beta=0.5, h=3.5 \mathrm{~m}$ : (a) flat bed conditions; (b) with ripple predictor switched on.

Fig. 4 shows the transport rate behaviour with $u_{\text {rms }}$ for a typical progressive surface wave. Note that any degree of acceleration skewness is omitted in this example, in order to enable comparison with the "equivalent" velocity-skewed oscillatory flow in Fig. 2. Comparison of Fig. 4a with Fig. 2a shows that the real wave effects lead to significantly larger transport rates for the medium sand, approximately by a factor 1.5 , while for the fine sands the effects lead to a change of direction of the net transport rate, in agreement, at least qualitatively, with the transport rates observed in the large wave flume experiments of Ribberink et al. (2000) and Schretlen et al. (2009). Roughly between 0.45 $<u_{\text {rms }}<0.65 \mathrm{~m} / \mathrm{s}$ it seems that the fine sand transport rate is constant, which appears to be the net effect of a decrease in transport due to offshore-directed phase-lag effects being in balance with the increase in transport related to the onshore-directed surface wave effects. Phase-lag effects related to ripples still lead to small negative transport rates for low orbital velocities in Fig. 4b, although experimental evidence for this behaviour still has to be obtained. It may be inferred from these results together with the behaviour shown in Fig. $3 \mathrm{~b}$ that the transport rates become positive throughout the entire $u_{\mathrm{rms}}$ range when a certain degree of acceleration skewness is present in the wave orbital velocity.

\section{Current Alone}

When no waves are present and a steady flow is the only driving mechanism for sand transport $(\alpha$ $=1$ ), the Shields parameter becomes the Shields parameter for current alone:

$$
\theta_{\delta}=\frac{\frac{1}{2} f_{\delta} u_{\delta}^{2}}{(s-1) g d_{50}}
$$

By applying Eq. 41 to the load formula (Eq. 15) the net transport rate in the direction of the current becomes:

$$
\Phi=\frac{q_{\mathrm{s}}}{\sqrt{(s-1) g d_{50}^{3}}}=\sqrt{\theta_{\delta}} \Omega_{\delta}
$$




\section{COMPARISON WITH MEASURED NET TRANSPORT RATES}

Table 1 presents an overview of the range of conditions covered by the database used for calibration of the new model. The database contains measured net transport rates for a wide range of full-scale conditions in both the rippled bed and sheet flow regime, including oscillatory flows with velocity skewness or acceleration skewness (or a combination of both), oscillatory flows with superimposed currents and non-breaking (shoaling) surface waves. The calibration procedure involved the following steps. First, the coefficients in the phase lag parameter $\alpha_{\mathrm{s}}$ and $\alpha_{\mathrm{r}}$ were tuned to obtain the highest correlation between measured and predicted transport rates for the sheet flow regime and rippled bed regime respectively. The proportionality constant $m$ was found from least-square fitting a straight line with zero intercept through all the measured and computed transport rates. This procedure was repeated for different values of the proportionality constant $n$. Next, the following coefficients were calibrated to obtain the best agreement between predictions and measurements for selected subdatasets: 1) the relative weight of the current friction factor and wave friction factor through coefficient $\sigma, 2$ ) the form roughness coefficient for rippled bed conditions $p, 3$ ) the coefficient for increased mobile bed roughness for fine sands $\mu$, and 4) for real wave conditions the level at which the vertical orbital is calculated through coefficient $\varepsilon$. Following the calibration of these data subsets the coefficients $m$ and $n$ were recalibrated using the entire database, resulting in $m=9.48$ and $n=1.2$ and phase-lag parameter coefficients $\alpha_{\mathrm{r}}=9.3$ and $\alpha_{\mathrm{s}}=8$.

\begin{tabular}{|c|c|}
\hline $\begin{array}{l}\text { Maximum velocity } u_{\max } \\
\text { Wave period } T \\
\text { Median Grain size } d_{50} \\
\text { Degree of velocity skewness } R \\
\text { Degree of acceleration skewness } \beta \\
\text { Net current velocity } u_{\delta}\end{array}$ & $\begin{array}{l}0.16-1.72 \mathrm{~m} / \mathrm{s} \\
4-12.5 \mathrm{~s} \\
0.13-0.46 \mathrm{~mm} \\
0.5-0.7 \\
0.5-0.8 \\
-0.44-+0.5 \mathrm{~m} / \mathrm{s}\end{array}$ \\
\hline $\begin{array}{l}\text { Number of velocity skewed flows } \\
\text { Number of acceleration skewed flows } \\
\text { Number of osc. flows with current } \\
\text { Surface wave conditions }\end{array}$ & $\begin{array}{l}92 \\
53 \\
50 \\
11 \\
\end{array}$ \\
\hline Total number of conditions & 206 \\
\hline
\end{tabular}

Fig. 5 shows the comparison between all 206 measured transport rates in the database and the predictions by the calibrated model. It is shown that for nearly all the conditions the correct transport direction is predicted, the exceptions being a few conditions near the origin for which the measured transport rates are very low (and the measurement errors are the largest). The majority of the conditions are predicted within a factor 2 of the measurements. The model performance for each of the subgroups is summarized in Table 2, which lists the number of conditions contained in each data subset, $N$, the correlation coefficient, $r$, and the percentages of the predictions falling within a factor 2 and factor 5 of the measurements. Overall very good agreement is obtained with $77 \%$ of the predictions falling within a factor 2 of the measurements. It was mentioned before that during the development and calibration of the model, we have constantly used the measured ripple dimensions as input to the model. Table 2 therefore also shows the performance of the model when the ripple dimensions are predicted using O'Donoghue et al.'s (2006) method. Obviously, incorporating a ripple predictor creates an extra source of uncertainty in the formula which is reflected in some reduction of the performance.

\begin{tabular}{|lcccc|}
\hline \multicolumn{5}{|l|}{ Table 2. Quantitative performance measures for the data subsets. } \\
\hline Data (sub)set & $N$ & $r$ & $\%$ fac 2 & $\%$ fac 5 \\
\hline Velocity skewed osc flows & 92 & 0.89 & 71 & 90 \\
Acceleration-skewed osc.flows & 53 & 0.91 & 79 & 98 \\
Osc. flows + current & 50 & 0.95 & 86 & 92 \\
Progressive surface waves & 11 & 0.39 & 82 & 100 \\
\hline All & 206 & 0.89 & 77 & 93 \\
All with ripple predictor & 206 & 0.88 & 65 & 85 \\
\hline
\end{tabular}




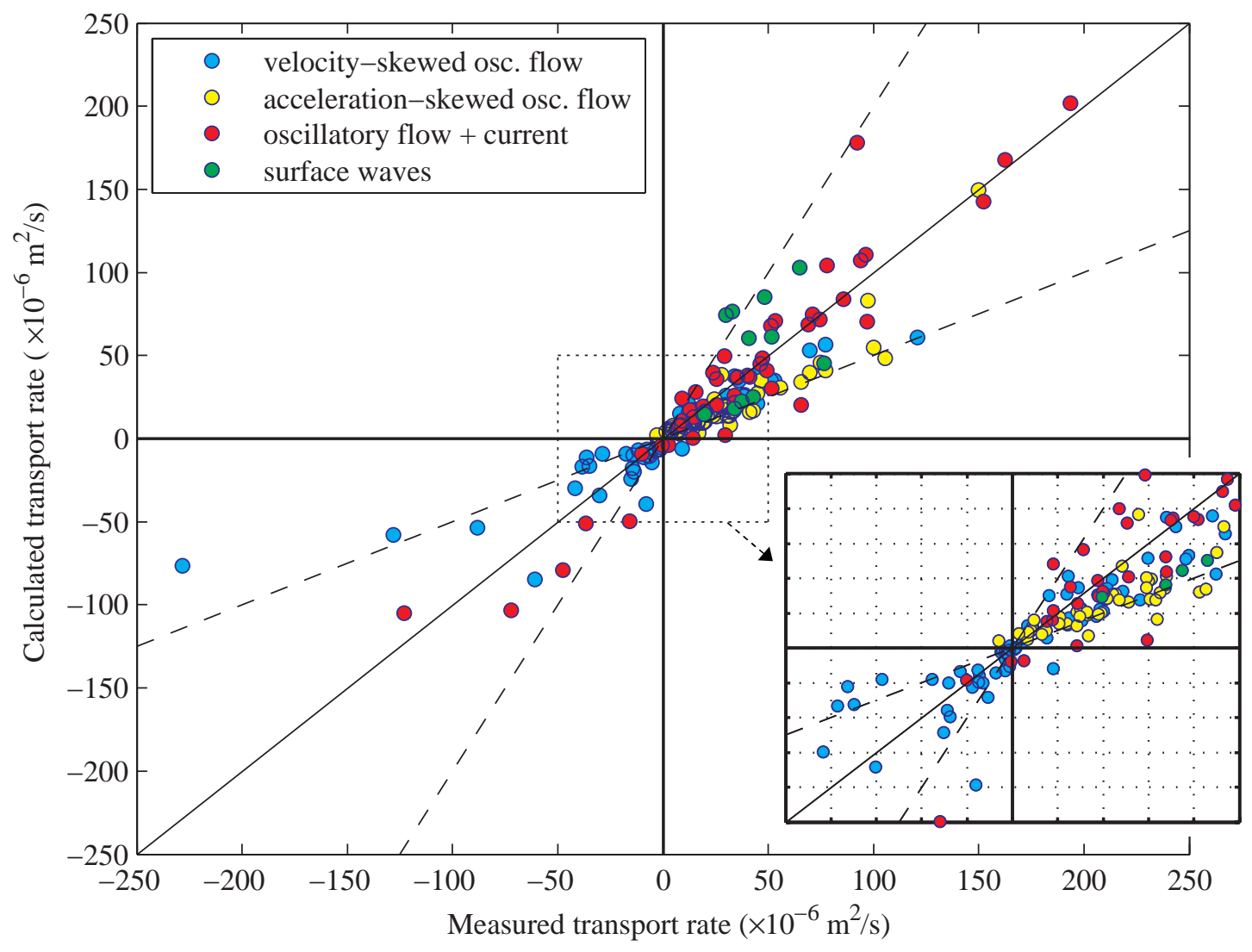

Figure 5: Model performance for all 206 conditions contained in the database. The solid line indicates perfect agreement, the dashed lines a factor 2 difference. The model uses measured ripple dimensions.

Finally, to test the performance of the calibrated model for steady flow transport rates, we have compared the model predictions with an independent dataset of bedload net transport rates for steady flows (Guy et al., 1966; Van den Berg, 1986; Nnadi and Wilson, 1992). The same datasets have been used by Ribberink (1998) for model development but were not used in the development or calibration of the present formula. The combined dataset comprises 137 conditions with current velocities ranging between $0.32-2.03 \mathrm{~m} / \mathrm{s}$ and median grain sizes between $0.19-3.8 \mathrm{~mm}$. The results and quantitative performance measures in Fig. 6 illustrate that the new formula also is quite capable of predicting the net transport rates in bedload dominated conditions for steady flows. In fact, these results are not entirely surprising since, for steady flows, the present formula is very similar to Ribberink's (1998) formula, which gave very good agreement for calibration coefficients $n=1.17$ and $m=10.4$.

\section{CONCLUSIONS}

A new practical sand transport model is developed for non-breaking waves, non-breaking waves with currents and currents alone. The model is developed and calibrated on the basis of a large dataset of measured net transport rates for a wide range of hydrodynamic and sand conditions typical for coastal areas.

The formula is semi-unsteady based on the half-wave cycle concept and accounts for the bedload and suspended load within the wave boundary layer in the rippled bed regime and sheet flow regime; for steady flow the model accounts for bedload only. Data and new insights from flow tunnel and large wave flume experiments conducted within the SANTOSS project have been used to include the effects of acceleration skewness and the effects related to progressive surface waves.

When the experimentally observed ripple dimensions are used, the new formula is able to predict $77 \%$ of the transport rates within a factor 2 of the measured transport rates, and $93 \%$ within a factor 5 


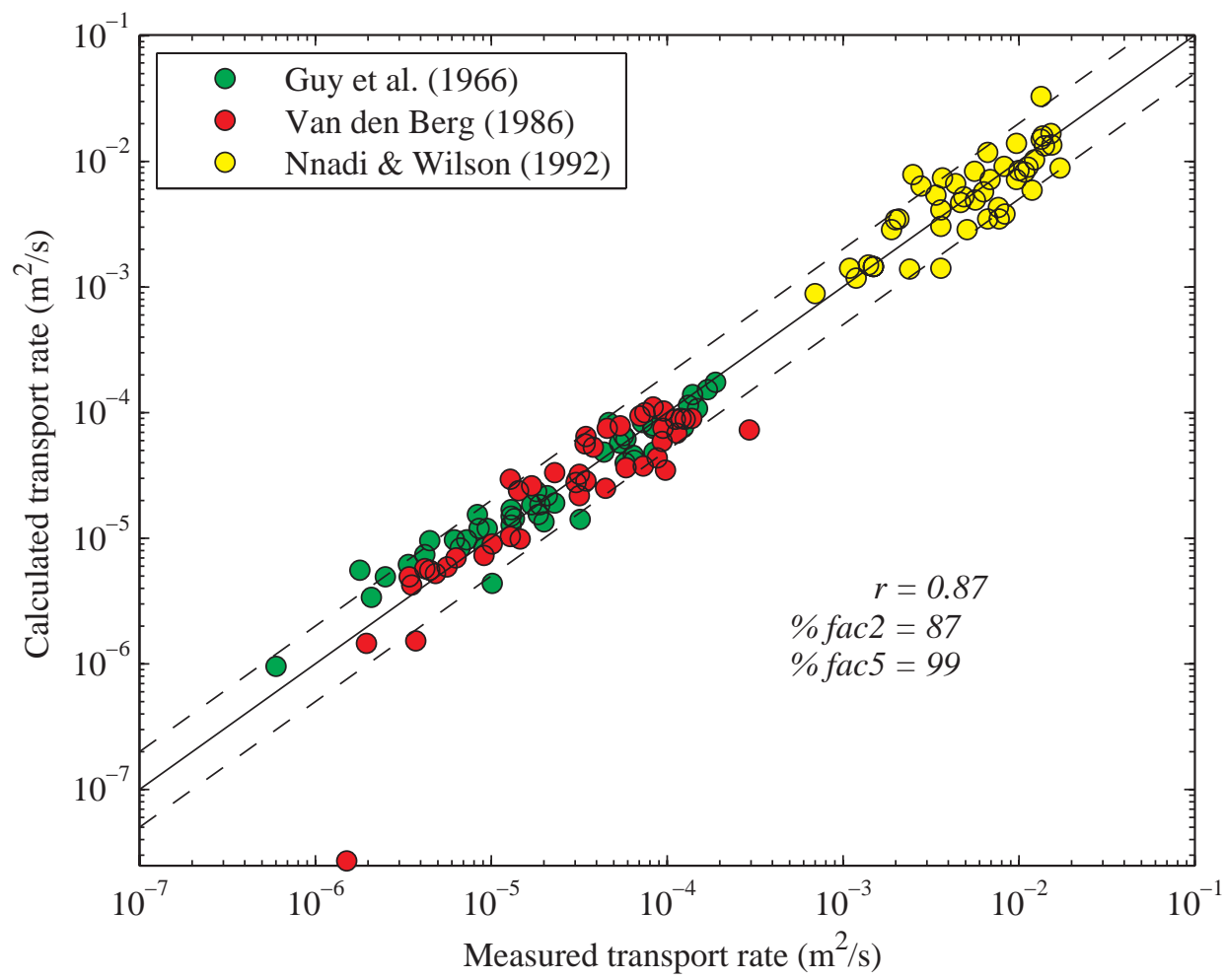

Figure 6: Model performance for steady flow conditions.

of the measurements. When applied in combination with a ripple predictor these figures reduce to $65 \%$ and $85 \%$ respectively. A comparison with an independent dataset of 137 bedload transport rates for a wide range of steady flow conditions resulted in $87 \%$ within a factor 2 and $99 \%$ within a factor 5 of the measurements.

The model is currently being implemented in a cross-shore morphological model to further test its validity and compare its performance with existing transport formulae. Future work is aimed at i) further improvement of modeling surface wave effects, and ii) extending the model to irregular wave and breaking wave conditions.

\section{ACKNOWLEDGMENTS}

This work is part of the SANTOSS project ('SANd Transport in OScillatory flows in the Sheetflow regime') funded by the UK's EPSRC (GR/T28089/01) and STW in The Netherlands (TCB.6586). The first author would also like to thank the Royal Academy of Engineering in the UK for the award of an International Travel grant to assist with ICCE 2010 attendance.

\section{REFERENCES}

Bailard, J.A., 1981. An energetics total load sediment transport model for a plane sloping beach, Journal of Geophysical Research, 86(C11), 10,938-10,954.

Dibajnia, M. and A. Watanabe. 1992. Sheet flow under nonlinear waves and currents, Proceedings of $23^{\text {rd }}$ International Conference on Coastal Engineering, ASCE, 2015-2028.

Dohmen-Janssen, C.M. 1999. Grain size influence on sediment transport in oscillatory sheet flow, phase lags and mobile-bed effects. Ph.D. thesis, Delft University of Technology, The Netherlands.

Elfrink, B., D.M. Hanes, and B.G. Ruessink. 2006. Parameterization and simulation of near bed orbital velocities under irregular waves in shallow water. Coastal Engineering, 53, 915-927.

Guy, H.P., D.B. Simons, and E.V. Richardson. 1966. Summary of alluvial channel data from flume experiments 1956-1961, US Geological Survey, Prof. Paper 462-I, Washington, DC.

Kranenburg, W.M., J.S. Ribberink, and R.E. Uittenbogaard. 2010. Sand transport by surface waves: can streaming explain the onshore transport? Proceedings of $32^{\text {nd }}$ International Conference on Coastal Engineering, Shanghai, China. 
Madsen, O.S., and W.D. Grant. 1976. Sediment transport in the coastal environment. MIT Ralph M. Parsons Lab., Rep. 209, Cambridge, USA.

O'Donoghue, T., J.S. Doucette, J.J. van der Werf, and J.S. Ribberink. 2006. The dimensions of sand ripples in full-scale oscillatory flows. Coastal Engineering, 53, 997-1012.

Nielsen, P. 1992. Coastal bottom boundary layers and sediment transport. Advanced Series on Ocean Engineering, vol. 4. World Scientific.

Nielsen, P. 2006. Sheet flow sediment transport under waves with acceleration skewness and boundary layer streaming, Coastal Engineering, 53, 749-758.

Nnadi, F.N., and K.C. Wilson. 1992. Motion of contact-load particles at high shear stress, Journal of Hydraulic Engineering. ASCE 118 (12).

Ribberink, J.S. 1998. Bed-load transport for steady flows and unsteady oscillatory flows. Coastal Engineering, 34, 59-82.

Ribberink, J.S., C.M. Dohmen-Janssen, D.M. Hanes, S.R. McLean and C. Vincent, 2000. Near-bed sand transport mechanisms under waves. Proceedings $27^{\text {th }}$ International Conference on Coastal Engineering. ASCE, pp. 3263-3276.

Ribberink, J.S., D.A. van der A, R.H. Buijsrogge. 2010. SANTOSS transport model - A new formula for sand transport under waves and currents. Report SANTOSS_UT_IR3, University of Twente, The Netherlands.

Schretlen, J.J.L.M., J.S. Ribberink, and T. O’Donoghue. 2009. Sand transport under full-scale surface waves. Proceedings of Coastal Dynamics 2009, World Scientific, paper 123.

Silva, P.S., T. Abreu, D.A. van der A, F. Sancho, B.G. Ruessink, J.J. van der Werf, and J.S. Ribberink. submitted. Sediment transport in non-linear skewed oscillatory flows: the Transkew experiments. Journal of Hydraulic Research.

Soulsby, R.L. 1997. Dynamics of marine sands, Thomas Telford Publications, London, pp. 272.

Swart, D.H. 1974. Offshore sediment transport and equilibrium beach profiles. Delft Hydraulics Laboratory Publication 131, Delft Hydraulics, The Netherlands.

Van den Berg, J.H. 1986. Aspects of sediment and morphodyanmics of subtidal deposits of the Oosterscehlde (the Netherlands), Rijkswaterstaat, Communications no. 43.

Van der A, D.A. 2010. Effects of acceleration skewness on oscillatory boundary layers and sheet flow sand transport. $\mathrm{PhD}$ Thesis, University of Aberdeen.

Van der A, D.A., T. O’Donoghue, A.G. Davies and J.S. Ribberink. 2008. Effects of acceleration skewness on rough bed oscillatory boundary layer flow. Proceedings of $31^{\text {st }}$ International Conference on Coastal Engineering, World Scientific, 1583-1595.

Van der A, D.A., T. O'Donoghue, and J.S. Ribberink. 2009. Sheet flow sand transport processes in oscillatory flows with acceleration skewness. Proceedings of Coastal Dynamics 2009, World Scientific, paper no 133 .

Van der A, D.A., T. O’Donoghue and J.S. Ribberink. 2010. Measurements of sheet flow transport in acceleration-skewed oscillatory flow and comparison with practical formulations, Coastal Engineering, 57, 331-342.

Van der Werf, J.J., J.S. Ribberink and T. O’Donoghue. 2007. Development of a new practical model for sand transport induced by non-breaking waves and currents. Coastal Sediments '07, ASCE, 42-55.

Van der Werf, J.J., J.J.L.M. Schretlen, J.S. Ribberink, and T. O’Donoghue. 2009. Database of fullscale laboratory experiments on wave-driven sand transport processes, Coastal Engineering, 56, 726-732.

Van Rijn, L.C. 2007. Unified view of sediment transport by currents and waves. I: Initiation of motion, bed roughness, and bed load transport. Journal of Hydraulic Engineering, 233(6), 649-667.

Wilson, K.C. 1989. Mobile-bed friction at high shear stress. Journal of Hydraulic Engineering, 115(6), 825-830. 